\title{
Giga-speed bioinformatics to power Genome Canada
}

Canada is implementing a national genomics initiative with the hope of thrusting the country into "genomics preeminence." Aided by the parallel development of the fastest bioinformatics network in the world, Genome Canada is expected to add over Cdn $\$ 1.5$ billion to the Canadian economy within five years. However, it remains to be seen whether a speedy network is enough to revive the country's genome effort.

According to Lap-Chee Tsui, cochair of Genome Canada, and one discoverer of the CFTR (cystic fibrosis transmembrane conductance regulator) gene, some $25 \%$ of the disease-related genes had been discovered in Canada or by Canadians until 1996, when Canadian Prime Minister Jean Chrétien, in a cost-cutting exercise to achieve a balanced budget, axed the Canadian Genome Analysis and Technology Program, the federal agency through which genomics research was funded in Canada. "The current reality is that sources of funding and systems of accountability have become highly diverse and increasingly fragmented," comments a Genome Canada official, "Canada is falling behind, not only through serious underfunding, but also by the lack of cohesive efforts."

In an effort to remedy this-and with a budget surplus-Chrétien has committed Cdn $\$ 250$ million in new money to Genome Canada covering 1999 to 2004. In addition, federal government health science, natural science, and social science granting councils have pledged $\$ 65$ million over the same period. The Canadian Foundation for Innovation is contributing $\$ 60$ million, and financial commitments from provincial governments, industry, and voluntary agencies total $\$ 125$ million to 2004 . The sum- $\$ 500$ million for five years-will enable Genome Canada "not to find the best back road by which to catch up, but the expressway by which to get ahead," comments Lap-Chee Tsui.

Genome Canada's mandate is to coordinate Canadian genomics programs into a network of centers to provide the platform technologies and knowledge required for further research. National research centers will receive from $\$ 40$ million in year 1 up to $\$ 65$ million by year 5 . Funding of targeted applications will increase annually from $\$ 15$ million in year 1 to $\$ 25$ million in year 5 . Training of skilled genomics technicians will occur throughout the project's life, with $\$ 3$ million allotted annually for a training pro-

Brian Hoyle is a science writer based in Halifax, Nova Scotia. gram. Targeted collaborative research and implementation will follow by the year 2004 .

In addition to the new funding, genomics researchers in Canada now have access to the Canadian Bioinformatics Resource (CBR; Halifax, Nova Scotia), the world's first gigabyte network. The CBR is expected to be a cornerstone of Canada's molecular biology and biotechnology research infrastructure, says Christoph Sensen, manager of the network. It utilizes the national high-speed $\mathrm{CA}^{\star}$ net II internet and comprises over 60 high-performance servers and workstations at six National Research Council of Canada centers nationwide (which originally funded the CBR), and the Rockfeller University in

\section{Some $25 \%$ of disease- related genes had been discovered in Canada or by Canadians until 1996, when Jean Chrétien axed the Canadian Genome Analysis and Technology in a cost- cutting exercise.}

New York. By the end of the year, current $\mathrm{CA}^{\star}$ net II links of 45 megabytes per second will all have been replaced by $\mathrm{CA}^{\star}$ net III links of 300 gigabytes per second.

"Nobody has ever set up anything like this before...we are a generation beyond everyone else because of the networking environment we can tap into," says Christoph Sensen, who says speed will be a major competitive advantage to Genome Canada. Once fully implemented, CBR should allow all users, wherever they are based, to access and decipher raw data from over 100 databases in real time, even if using video streaming, he says.

Harry Mangalam, CEO of tacg Informatics (Irvine, CA) sees other benefits. " $[\mathrm{CBR}]$ would increase the pipeline for research data transfer, encouraging more services to spring up," he says. "Since you can push more data, more network/informatics research services could be made accessible to more researchers across [Canada]. Tacg Informatics is a bioinformatics consulting and software development company currently implementing a public gene expression database for the National Center for Genome Resources in Santa Fe, New Mexico.

The CBR model of networked geographically distinct centers builds on the successes of the UK, the European, and Swiss bioinformatics efforts. "One advantage to this network is that it will make Canadian computing resources more attractive than ones outside of Canada because of the bandwidth considerations," says Mangalam.

Amos Bairoch, SWISS-PROT group leader of the Swiss Institute of Bioinformatics, agrees that "CBR's network speed does make it truly unique from other bioinformatics networks." In August, CBR was selected as the sole North American mirror site of the ExPASy server of the Swiss Institute of Bioinformatics. "Any serious bioinformatics center, and the CBR is certainly one of them, makes a big impact on both the academic and industrial life science research environment of a given country. I do not see why it will not be the case in Canada."

However, the CBR model differs considerably from the successful centers in the US. The National Institutes of Health and National Science Foundation "have never insisted on Centers of Excellence," says Mangalam. "They allow good research proposals to be funded wherever they are, and the adjoining institutes and organizations all benefit," he says, "What has made US biotech and bioinformatics so vibrant was the ability of small companies to start up very easily to exploit an idea, and then either grow or go bust based on their potential." CBR manager Christoph Sensen acknowledges that and says CBR reflects the realities of Canadian science. "Compared to the US, Canada's science blanket is thin, but we know how to network."

"It really does seem that Canadian biotechnology and bioinformatics is lagging behind what it could do," says Harry Mangalam. "It will be interesting to see if this approach yields the results that they want or whether it will take a fundamentally different approach to encourage development of a thriving homegrown bioinformatics community."

Meanwhile, the government anticipates these new investments to establish Genome Canada and the CBR to reap benefits for the Canadian economy. Genome Canada estimates that its genomics strategy will generate over Cdn $\$ 1.5$ billion within five years from resulting genomics companies, genomicsrelated jobs, and breakthroughs in genomics research. And CBR's bioinformatics training initiative, underway in collaboration with the Canadian Genetic Diseases Network and the Biotechnology Human Resources Council, will provide bioinformaticians for the genomics-driven industries.

Brian Hoyle 